\title{
Factores de Riesgo para el Desarrollo de Complicaciones en las Toracostomías Cerradas por Trauma
}

\author{
Rocío I Díaz, Rafael Andrade-Alegre
}

\section{RESUMEN}

Introducción: La toracostomía cerrada es un procedimiento quirúrgico considerado sencillo y ampliamente utilizado en pacientes con trauma torácico. Al ser un procedimiento realizado a ciegas, posee mayor probabilidad de complicaciones. En nuestro hospital se colocan alrededor de 315 tubos de tórax por trauma al año. El objetivo de nuestro estudio es determinar, de manera prospectiva, los factores de riesgo para el desarrollo de complicaciones en las toracostomías cerradas por causas traumáticas.

Metodología: Se trata de un estudio prospectivo, observacional tipo cohorte, de corte longitudinal. Durante un periodo de 7 meses, se incluyeron pacientes consecutivos con diagnóstico de trauma torácico cerrado o penetrante y a los que se les colocó un tubo de tórax por esta causa. La muestra consistió de 152 casos. Los datos se recolectaron utilizando el expediente clínico. Las variables fueron analizadas con un nivel de confianza del $95 \%$.

Resultados: Predominó el sexo masculino (86.8\%) y la edad promedio fue de 29 años. Un $37.5 \%$ de los pacientes presentó un Injury Severity Score mayor o igual a 16. Cuarenta y dos pacientes tuvieron algún tipo de complicación, lo que representa un $27.6 \%$ de la muestra. De este grupo, solo 14 pacientes requirieron un segundo drenaje pleural o una toracotomía abierta. La presencia de hemotórax resultó estadísticamente significativa para el desarrollo de complicaciones $(p=0.041)$, al igual que la incorrecta colocación del tubo de tórax $(p=0.006)$.

Conclusión: La toracostomía cerrada por trauma tiene una significativa morbilidad. La presencia de hemotórax es un factor de riesgo estadísticamente significativo para el desarrollo de complicaciones al igual que la incorrecta colocación del tubo de tórax. Proponemos la elaboración de un estudio prospectivo utilizando el ultrasonido como guía para la adecuada colocación del tubo de tórax y así determinar su utilidad en la disminución de complicaciones.

Palabras claves: Tubo de tórax, Toracostomía cerrada, Trauma, Complicaciones, Factores de riesgo.

\section{ABSTRACT}

Introduction: Tube thoracostomy is a surgical procedure considered simple and widely used in patients with thoracic trauma. Being a blind procedure, it has a larger probability of complications. In our hospital, around 315 chest tubes are inserted per year due to traumatic causes. The objective of this study is to determine, in a prospective way, the risk factors for developing complications due to chest tube insertion in chest trauma patients.

Methods: This is a prospective, observational, cohort type and longitudinal study. During a 7 months period, all patients with diagnosis of blunt or penetrating chest trauma to whom a chest tube was inserted were included. Total sample consisted of 152 cases. Data was collected using the clinical file. Variables were analyzed with a level of confidence of $95 \%$.
Results: Male sex was $86.8 \%$ and average age was 29 years. $35.7 \%$ of the patients had an Injury Severity Score of 16 or more. Forty-two patients $(27.6 \%)$ presented some sort of complication. Only 14 patients required a second chest tube or open thoracotomy. Hemothorax was statistically significant for the development of complications $(p=0.041)$, as it was the misplacement of the chest tube $(p=0.0006)$.

Conclusion: Tube thoracostomy for trauma has a significant morbidity. Presence of hemothorax and misplacement of chest tube are risk factors statistically significant for the development of complications. We propose a prospective study using ultrasound as a guide for the correct placement of chest tube and in this way determine whether this procedure will be helpful in diminishing complications.

Keywords: Chest tube, Tube thoracostomy, Trauma, Complications, Risk factors.

How to cite this article: Díaz RI, Andrade-Alegre R. Factores de Riesgo para el Desarrollo de Complicaciones en las Toracostomías Cerradas por Trauma. Panam J Trauma Critical Care Emerg Surg 2013;2(2):69-73.

Source of support: Nil

Conflict of interest: None

\section{INTRODUCCIÓN}

La toracostomía cerrada es un procedimiento quirúrgico considerado sencillo y es ampliamente utilizado en pacientes de trauma. En nuestro hospital se colocan alrededor de 315 tubos de tórax (TT). Por tratarse de un procedimiento realizado a ciegas, posee mayor probabilidad de complicaciones. ${ }^{1}$ La tasa global de complicaciones relacionadas a este procedimiento puede ser hasta un 30\%.,

La mayoría de los estudios relacionados a las complicaciones de (TT) han sido realizados de manera retrospectiva. ${ }^{1-4}$ El propósito de nuestra investigación es determinar de manera prospectiva los factores de riesgo para el desarrollo de complicaciones en las toracostomías cerradas por causas traumáticas.

\section{METOdOLOGÍA}

Se trata de un estudio prospectivo, observacional tipo cohorte, de corte longitudinal. Durante un periodo de 7 meses (1/6/10-31/12/10), se incluyeron pacientes consecutivos con diagnóstico de trauma torácico contuso o penetrante, a los que se les colocó un TT, ya fuera dentro de nuestro hospital o referidos de otras instituciones. Se excluyeron pacientes 
menores de 15 años, aquéllos que fallecieron en menos de 24 horas, los que necesitaron toracotomía abierta en un periodo menor de 24 horas por causas no relacionadas al TT y los TT colocados por causas no traumáticas como empiemas, neumotórax espontáneos o eventos iatrogénicos traumáticos. La muestra final consistió de 152 pacientes.

Se diseñó un formulario para recolectar los datos del expediente clínico. Los pacientes fueron observados regularmente hasta su egreso hospitalario y se les dio un seguimiento de al menos 4 semanas en consulta externa. Las radiografías y las tomografías computadas fueron evaluadas por los autores, para determinar si había algún tipo de complicación posicional o post retiro del TT.

Por norma en nuestro hospital, la técnica para la colocación del TT es la recomendada por el ATLS. ${ }^{5}$ Los TT se extrajeron a discreción del equipo médico tratante, cuando el drenaje no fue activo, menor de $100 \mathrm{ml}$ en 24 horas y sin evidencia de fuga aérea.

Las variables estudiadas están resumidas en la Tabla 1. Se consideró que un TT estuvo retrasado cuando se colocó después de una hora de haber llegado el paciente al hospital. Igualmente se consideró que se administraron antibióticos de manera profiláctica cuando fueron utilizados justo antes de la colocación del drenaje pleural o dentro de la primera hora de su colocación.

El índice ISS de cada paciente se calculó manualmente y se agruparon a los pacientes en tres categorías: leve (ISS = 1-8), moderado (ISS $=9-15)$ y severo (ISS $\geq 16$ ).

Generalmente en la literatura las complicaciones por TT se han clasificado en aquellas por inserción, posicionales e infecciosas, ${ }^{1,2} \sin$ embargo, nosotros las dividimos en cuatro tipos: asociadas a la inserción del TT, posicionales, infecciosas y post retiro del TT. Pensamos que esta clasificación es más completa y más ilustrativa de las diferentes complicaciones.

Definimos las complicaciones relacionadas a la inserción del TT, como aquéllas en las que hubo laceración de la arteria intercostal, colocación dentro del parénquima pulmonar con perforación del pulmón y lesión de grandes vasos o corazón. A través del análisis de estudios de imágenes se definió si existió una complicación posicional cuando el TT fue colocado muy bajo en la caja torácica o se encontraba acodado, el orificio centinela se encontraba en el tejido subcutáneo o el TT se encontraba intercisural. Todas las radiografías y tomografías de tórax de cada paciente fueron analizadas. Se consideró como complicación infecciosa cuando el paciente presentó síntomas y signos de infección local o si en algún momento desarrolló un empiema. Las complicaciones relacionadas al retiro del TT, fueron aquéllas en las que hubo un neumotórax residual o persistencia de hemotórax/efusión en la radiografía post-extubación.
La información recolectada manualmente en los formularios fue trasladada a una base de datos utilizando el programa SPSS versión 17.0. Se utilizó un nivel de confianza del $95 \%$ y se consideró que un resultado fue estadísticamente significativo cuando se obtuvo un valor de $\mathrm{p}<0.05$.

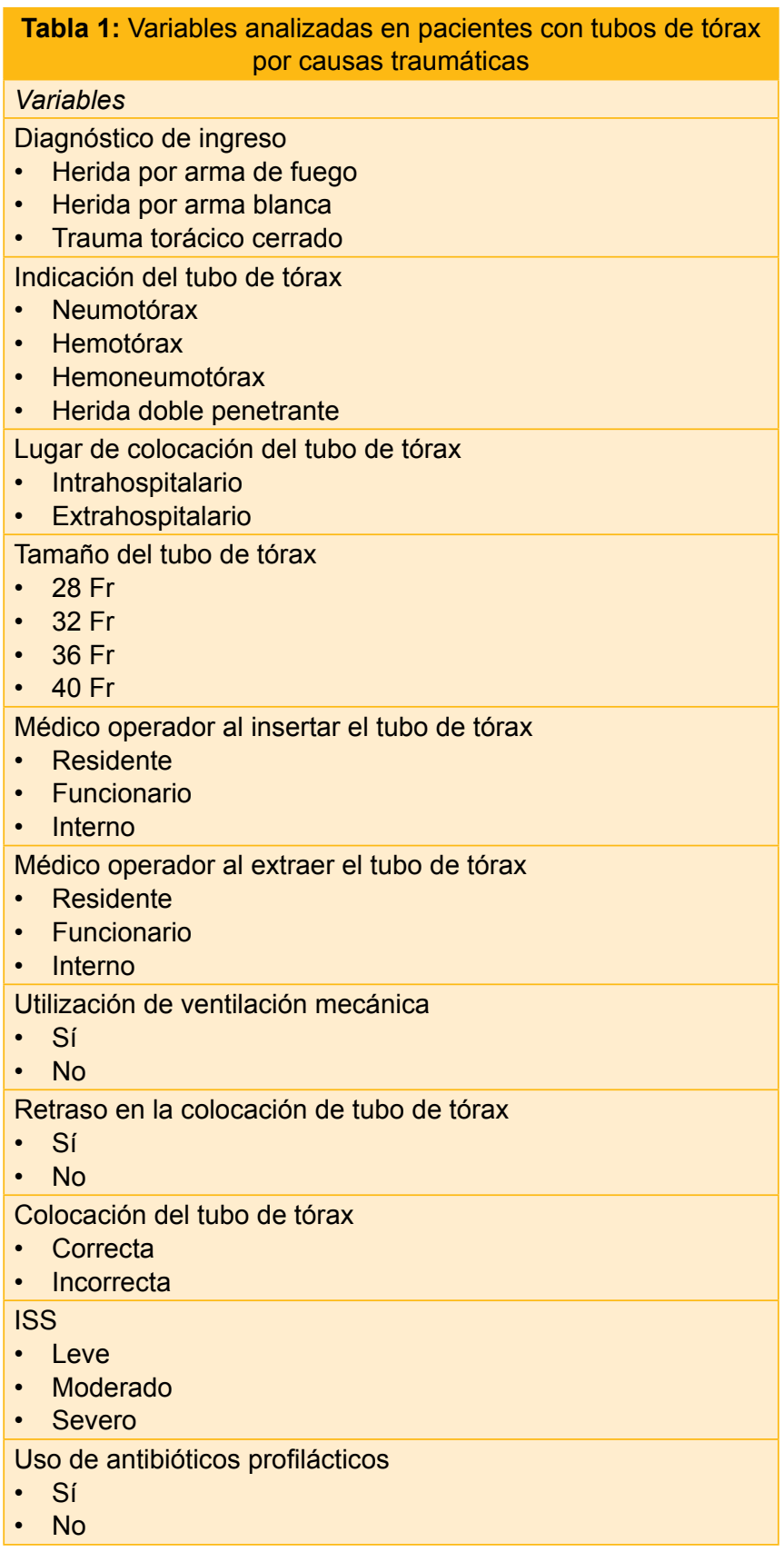

\section{RESULTADOS}

Un resumen de los resultados está representado en la Tabla 2. El $86.8 \%$ de los pacientes pertenecen al sexo masculino y $13.2 \%$ al femenino. La edad promedio fue 29 años con un rango entre 15 y 60 años.

Los diagnósticos de ingreso de los pacientes fueron: herida por arma de fuego (HPAF) en $44.1 \%$ de los casos, seguido de heridas por arma blanca (HPAB) en $36.8 \%$ y los traumas torácicos cerrados en un $17.8 \%$ (en 2 expedientes 
no se especificó el tipo de trauma). No hubo diferencia estadísticamente significativa entre los diagnósticos de ingresos en cuanto a complicaciones. Al agrupar las HPAF y las HPAB como trauma penetrantes y compararlas con los traumas torácicos cerrados, tampoco se encontró diferencia estadísticamente significativa en cuanto a aparición de complicaciones $(\mathrm{p}=0.664)$.

\begin{tabular}{|l|c|}
\hline $\begin{array}{c}\text { Tabla 2: Variables y su resultado estadístico en pacientes con } \\
\text { tubo de tórax debido a causas traumáticas }\end{array}$ \\
\hline Variable & Valor de $p$ \\
\hline Diagnóstico de Ingreso & 0.836 \\
\hline Indicación del tubo de tórax (hemotórax) & 0.041 \\
\hline Sala & 0.125 \\
\hline Tamaño del tubo de tórax & 0.493 \\
\hline Médico operador al insertar el tubo de tórax & 0.796 \\
\hline Médico operador al extraer el tubo de tórax & 0.523 \\
\hline Utilización de ventilación mecánica & 0.228 \\
\hline Retraso en la colocación de tubo de tórax & 0.651 \\
\hline Colocación del tubo de tórax (incorrecta) & 0.006 \\
\hline ISS & 0.227 \\
\hline Uso de antibióticos profilácticos & 0.949 \\
\hline
\end{tabular}

La principal indicación por la que se colocó el TT fue por hemotórax (36.2\%), seguido por hemoneumotórax (21.7\%), neumotórax $(17.1 \%)$ y heridas doble penetrantes $(9.9 \%)$. Hubo un $15.1 \%$ de pacientes en los que no se especificó la razón de la colocación del TT. Los pacientes a los que se les colocó el TT debido a un hemotórax o un hemoneumotórax, presentaron mayor cantidad de complicaciones $(\mathrm{p}=0.041)$ en comparación con aquellos a los que se les colocó por tener un neumotórax o una herida doble penetrante.

Dentro del hospital, la mayoría de los TT (58.6\%) fueron colocados en el servicio de urgencias, seguido de un $21.7 \%$ de casos colocados en otras instituciones de salud. La colocación de TT fuera de nuestra institución no demostró diferencias estadísticamente significativas en cuanto a la aparición de complicaciones $(\mathrm{p}=0.125)$ al compararlos con nuestros casos.

El drenaje pleural tuvo una duración promedio de 8.44 días en pacientes complicados versus 5.20 días en pacientes no complicados. Con respecto al tamaño del TT, la mayoría de los cirujanos utilizaron drenajes calibre $32 \mathrm{Fr}$ (44\%) dentro de un rango de 28-40 Fr. No hubo diferencias estadísticamente significativas $(\mathrm{p}=0.493)$ entre el tamaño del TT y las complicaciones presentadas.

En cuanto al médico que inserta el TT, se encontró que el $61.8 \%$ de los casos fueron colocados por un médico residente, seguido de un $28.3 \%$ de médicos funcionarios. El 10\% restante fueron pacientes sin datos, es decir, no se especificó en el expediente quién colocó el TT y dos TT fueron colocados por médicos internos. No se encontró diferencia estadísticamente significativa en cuanto a la aparición de complicaciones según el médico que insertó el TT $(p=0.796)$. El $42.8 \%$ de los TT fue extraído por médicos residentes. Igualmente, el porcentaje restante fueron pacientes sin datos, o médicos internos.

El $17.8 \%$ de los pacientes ameritaron ventilación mecánica y en $28.9 \%$ de los casos, los TT se consideraron retrasados. Sin embargo, ninguna de estas dos variables se relacionó con mayor aparición de complicaciones.

El $37.5 \%$ de los casos presentaron ISS severos, 32.9\% moderados y $29.6 \%$ leves. No se encontraron diferencias estadísticamente significativas en cuanto a la severidad del ISS y la aparición de complicaciones debidas al TT.

A la mayoría de los casos $(65.1 \%)$ no se les colocó antibióticos profilácticos. En los que se usó fue a discreción del cirujano. No hubo diferencias estadísticamente significativas en cuanto al uso o no de antibióticos profilácticos y la aparición de complicaciones $(\mathrm{p}=0.949)$.

Cuarenta y dos pacientes tuvieron algún tipo de complicación, lo que representa un $27.6 \%$ de los casos estudiados. Este porcentaje se puede subdividir a su vez en complicaciones posicionales $45.2 \%$, post-retiro $40.5 \%$, infecciosas $11.9 \%$ y por inserción $2.53 \%$.

En relación al retiro de los TT, 103 (67.8\%) pacientes presentaron radiografías post-retiro normales, $16(10.5 \%)$ carecen de radiografía control post-retiro y 33 tuvieron hallazgos que variaron entre neumotórax residual, efusión residual, contusión o empiema.

De los 42 casos considerados como complicados, solo 14 requirieron un procedimiento adicional (segundo TT o toracotomía abierta), el resto de los pacientes fueron manejados con tratamiento conservador con seguimiento en la consulta externa (Tabla 3).

\begin{tabular}{|l|c|c|c|c|}
\hline \multicolumn{5}{|c|}{ Tabla 3: Tipos de complicaciones de los tubos de tórax y } \\
manejo correspondiente \\
Complicación & \multicolumn{4}{|c|}{ Tratamiento } \\
\cline { 2 - 5 } & Conservador & $\begin{array}{c}\text { Toracotomía } \\
\text { abierta }\end{array}$ & $\begin{array}{c}\text { Segundo } \\
\text { TT }\end{array}$ & Total \\
\hline Infecciosa & 3 & 1 & 1 & 5 \\
\hline Inserción & 1 & 0 & 0 & 1 \\
\hline Posicional & 12 & 4 & 3 & 19 \\
\hline Post-retiro & 12 & 0 & 5 & 17 \\
\hline Total & 28 & 5 & 9 & 42 \\
\hline
\end{tabular}

No hubo mortalidad asociada al TT. Fallecieron 3 pacientes con ISS severo: el primero por lesión neurológica severa por proyectil de arma de fuego; el segundo, por trauma craneoencefálico severo y politraumatismo secundario a atropello y el tercero, por trauma raquimedular a nivel de C2-C3 por proyectil de arma de fuego.

\section{DISCUSIÓN}

Nuestro estudio obtuvo un $27.6 \%$ de complicaciones relacionadas al TT, valor que se encuentra dentro de los 
rangos reportados en la literatura. ${ }^{1,2,6}$ Sin embargo, si consideramos que los verdaderos pacientes complicados fueron aquéllos que ameritaron un procedimiento adicional (segundo TT o toracotomía abierta), esta cifra se reduce dramáticamente a un $9.2 \%(14 / 152)$.

Nuestro protocolo definió como complicación postretiro aquellos pacientes que presentaron neumotórax o hemotórax/efusión residual en la radiografía de tórax postextubación; no obstante, la mayoría de estos casos fueron manejados de manera conservadora. En nuestra estadística, dentro del grupo de 17 pacientes con una complicación post-retiro, sólo 5 pacientes requirieron un segundo TT; y luego del seguimiento en consulta externa, ninguno requirió procedimiento adicional (Tabla 3).

Los factores que resultaron estadísticamente significativos para el desarrollo de complicaciones fueron la presencia de hemotórax y la inadecuada colocación de los TT. Existen reportes que demuestran que el hemotórax es un factor de riesgo para el desarrollo de complicaciones. ${ }^{7-9}$ En nuestro estudio, a los pacientes que se les colocó un TT debido a un hemotórax o a un hemoneumotórax, tuvieron un mayor riesgo de desarrollar una complicación posicional (drenaje inadecuado).

La inadecuada colocación de los TT se debe principalmente a que la toracostomía cerrada es un procedimiento que se realiza a ciegas y en tal sentido el TT puede quedar excluido limitando la adecuada evacuación de aire $y / o$ sangre.

En nuestro estudio no se encontraron otras variables con resultados estadísticamente significativos para el desarrollo de complicaciones. Es importante mencionar que a diferencia de otros estudios, ${ }^{1,6}$ no encontramos diferencia en cuanto al médico operador. Ni el nivel de entrenamiento (residente o funcionario) ni la especialidad (quirúrgica o urgencias) afectaron los resultados. No obstante, es necesario resaltar la importancia de un entrenamiento adecuado en la técnica de la toracostomía cerrada para evitar en lo posible las complicaciones.

El uso de antibióticos de manera profiláctica en la colocación de TT ha sido un tema de amplio debate y poco consenso. La controversia continúa: hay reportes que sugieren que el uso profiláctico de antibióticos disminuye las probabilidades de complicaciones infecciosas como lo son los meta-análisis de Sanabria y col. ${ }^{10}$ y Bosman y col. ${ }^{11}$ Sin embargo, al igual que otros autores, ${ }^{12,13}$ nuestro estudio no demostró que el uso profiláctico de antibióticos fuera un factor protector.

Durante la elaboración de nuestro estudio, incluimos el calibre del TT como una variable que hipotéticamente podría estar relacionada a mayores complicaciones en pacientes con calibres pequeños. $\mathrm{Al}$ igual que otros autores, ${ }^{14}$ nosotros encontramos que el calibre del TT no resultó ser un factor de riesgo para complicaciones.

La fortaleza de nuestro estudio radica en su carácter prospectivo, lo significativo de la muestra, los criterios radiográficos y el seguimiento de los casos. La mayor limitación es su carácter observacional pues los autores no pudimos intervenir ni estandarizar el manejo de los casos. Un ejemplo en este sentido fue el uso de antibióticos a discreción del médico tratante, sin la utilización de un protocolo.

\section{CONCLUSIÓN}

La toracostomía cerrada por trauma tiene una significativa morbilidad. La presencia de hemotórax es un factor de riesgo estadísticamente significativo para el desarrollo de complicaciones al igual que la incorrecta colocación del TT. En tal sentido, proponemos la elaboración de un estudio prospectivo aleatorio utilizando el ultrasonido como guía para la adecuada colocación del TT y así determinar su utilidad en la disminución de complicaciones.

\section{AGRADECIMIENTOS}

Agradecemos la valiosa contribución del Lic. Ricaurte Tuñón, Magister en Bioestadística e Investigación.

\section{REFERENCIAS}

1. Ball CG, Lord J, Laupland KB, Gmora S, Mulloy RH, Ng AK, et al. Chest tube complications: how well are we training our residents? Can J Surg 2007;50:450-458.

2. Bailey RC. Complications of tube thoracostomy in trauma. J Accid Emerg Med 2000;17:111-114.

3. Menger R, Telford G, Kim P, Bergey MR, Foreman J, Sarani B, et al. Complications following thoracic trauma managed with tube thoracostomy. Injury 2012;43:46-50.

4. Etoch SW, Bar-Natan MF, Miller FB, Richardson JD. Tube thoracostomy. Factors related to complications. Arch Surg 1995;130:521-525.

5. American College of Surgeons, Committee on Trauma. Advanced trauma life support course for doctors instructor course manual. Chicago, American College of Surgeons. Octava edición 2008;91-120.

6. Deneuville M. Morbidity of percutaneous tube thoracostomy in trauma patients. Eur J Cardiothorac Surg 2002;22:673-678.

7. DuBose J, Inaka K, Demetriades D, Scalea TM, O'Connor $\mathrm{J}$, Manaker J, et al. Management of post-traumatic retained hemothorax: a prospective, observational, multicenter AAST study. J Trauma Acute Care Surg 2012;72:11-22.

8. Salcedo A, Henao CA, Cardona A, Naranjo S, Villegas MI, Morales CH. Factores de Riesgo para Desarrollar Empiema Postraumático. Pan J Trauma Crit Care Emerg Surg 2013;1:163167.

9. Ramanathan R, Wolfe LG, Duane TM. Initial suction evacuation of traumatic hemothoraces: a novel approach to decreasing 
chest tube duration and complications. Am Surg 2012;78: 883-887.

10. Sanabria A, Valdivieso E, Gómez G, Echeverry G. Prophylactic antibiotics in chest trauma: a meta-analysis of high-quality studies. World J Surg 2006;30:1843-1847.

11. Bosman A, de Jong MB, Debeij J, van den Broek PJ, Schipper IB. Systematic review and meta-analysis of antibiotic prophylaxis to prevent infections from chest drains in blunt and penetrating thoracic injuries. Br J Surg 2012;99:506-513.

12. Maxwell RA, Campbell DJ, Fabian TC, Croce MA, Luchette FA, Kerwin AJ. Use of presumptive antibiotics following tube thoracostomy for traumatic hemopneumothorax in the prevention of empyema and pneumonia - a multi-center trial. J Trauma 2004;57:742-748.

13. Moore FO, Duane TM, Hu CK, Fox AD, McQuay N Jr, Lieber ML, et al. Presumptive antibiotic use in tube thoracostomy for traumatic hemopneumothorax: an Eastern Association for the Surgery of Trauma practice management guideline. J Trauma Acute Care Surg 2012;73:S341-344.
14. Inaba K, Lustenberger T, Recinos G, Georgiou C, Velmahos $\mathrm{GC}$, Brown $\mathrm{C}$, et al. Does size matter? A prospective analysis of 28-32 versus 36-40 French chest tube size in trauma. J Trauma Acute Care Surg 2012;72:422-427.

\section{ACERCA DE LOS AUTORES}

\section{Rocío I Díaz}

Intern, Sección de Cirugía Torácica, Santo Tomás Hospital, Ciudad de Panamá, República de Panama

\section{Rafael Andrade-Alegre}

Columbus College of Medicine and Health, Panamá, Republic of Panama

Correspondencia: Chief Thoracic Surgery Section, Santo Tomás Hospital, Columbus College of Medicine and Health Sciences, Panamá Republic of Panamá, Phone: (507)5075600, Fax: (507)2296646 e-mail: toravasc@cwpanama,net 